\title{
Test Platform for Electromagnetic Field Strength Measurements near Wind Energy Converting Systems
}

\author{
Sebastian Koj ${ }^{* 1}$, Petr Akhlamov', Jochen Bredemeyer ${ }^{2}$, Heyno Garbe ${ }^{1}$ \\ ${ }^{1}$ Gottfried Wilhelm Leibniz Universität Hannover, Institute of Electrical Engineering and Measurement \\ Technology, Department of Electromagnetic Compatibility, Appelstr. 9a 30167 Hannover, Germany, \\ 2 FCS Flight Calibration Services GmbH, Hermann-Blenk-Straße 32A, 38108 Braunschweig, Germany \\ *koj@geml.uni-hannover.de
}

\begin{abstract}
This paper presents a measuring concept for characterization of the electromagnetic fields near to wind energy converting systems (WECS) using a flying measuring platform (FMP). The FMP is based on an octocopter which includes a signal digitizer board and a receiver front end. The design and the realization of the receiver front end are described in this work. The main goal of the design is compact and lightweight equipment, which can be mounted on the FMP. The FMP can be started at any position with respect to the wind farm and climb to an altitude up to 500 meters. Furthermore, the developed receiver front end shows high performance in terms of dynamic range and selectivity.
\end{abstract}

Key words: wind energy converting systems, flying measuring platform, receiver front end, wind farm

\section{Introduction}

Motivated by the objectives of the Paris Agreement of 2015, all 195 participating states, including all EU members, are striving to reduce their $\mathrm{CO}_{2}$ emissions [1]. One approach to achieve this ambitious goal is the conversion of the energy extraction strategies from fossil to renewable sources, e.g. by using wind energy converting systems (WECS). Contrary to conservative energy sources, WECS are decentralized energy units, which are set up in windy regions, outside residential areas. In the search for a suitable installation location, areas close to airports or installations of terrestrial air navigation systems (TANS) come often into consideration. If WECS should be installed near the aforementioned infrastructure, the potential interactions between the WECS and the TANS must be considered.

One aspect is the unwanted electromagnetic (EM) emissions of the WECS, which can interfere the signals of the TANS. In order to determine the impact of the EM emissions of WECS, the current standardization [2], [3] and [4] merely prescribe measurements of the electric and magnetic field strength on ground level, at a distance of 30 meters from WECS. Due to the heights of modern WECS of more than 100 meters, the uncertainty, that the emissions emanating from the nacelle of a wind turbine are not recorded and are not assessed, arises. This fact increases the risk of disruption of TANS and is unacceptable.

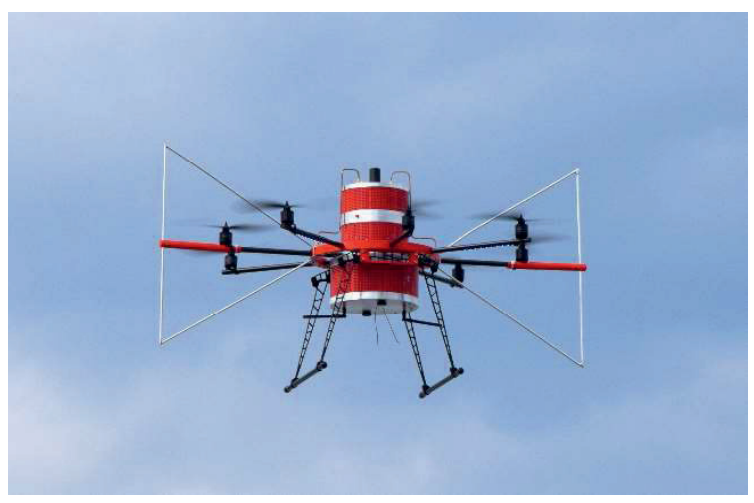

Fig. 1. Flying measurement platform (FMP). Photo: Marius Mihalachi, PTB

A further interaction mechanism, which has to be considered between WECS and TANS is due to unwanted reflections of TANS signals at the WECS. These reflected signals lead to incorrect information regarding the position of the aircraft and significantly influence the flight navigation. With both sketched interactions, the desire for a measurement of EM fields near the WECS at the level of the nacelle becomes obvious. When the $\mathrm{EM}$ emissions are measured, the disturbances at the nacelle level also can be recorded and evaluated. By measuring the signals of TANS in front and behind WECS, its impact on the transmission channel of the TANS signals can be examined. The presented metrological challenges can be mastered by using an octocopter as a measurement equipment carrier platform (Fig. 1). In addition to very good 
positioning accuracy and reproducibility, this approach offers the possibility to carry out measurements also within wind farms. The limited carrying capacity of an octocopter makes the use of commercially available measuring receivers infeasible due to their typical mass exceeding 20 kilograms. For this reason, a modularly designed flying measurement platform (FMP) shown in Fig. 1 has been developed [5], [6].

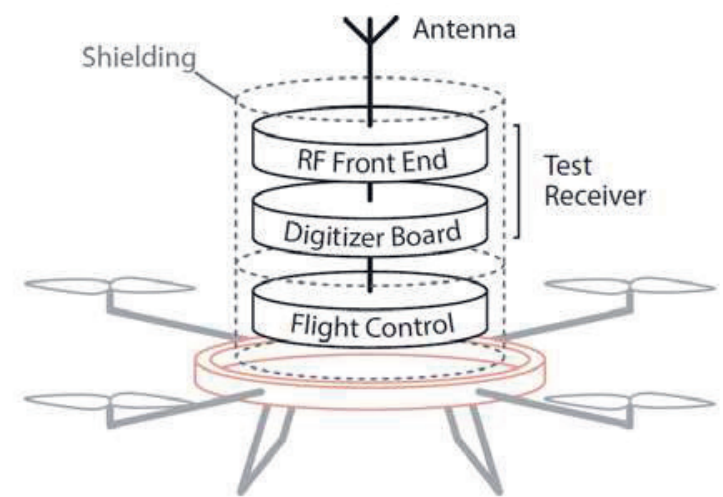

Fig. 2. Concept of the flying measurement platform (FMP).

As shown in Fig. 2, the FMP consists of a flight control unit, a signal digitizer board and a receiver front end with an antenna. The signal digitizer board carries out basic signal processing and controls the storage of data. In order to guarantee the precision of current position of FMP, the flight control board is used. Since the FMP is modularly designed, it can be easily equipped with different types of radio frequency receiver front ends (RF front end). These can be optimized for different frequency ranges, especially for the range from $150 \mathrm{kHz}$ until $30 \mathrm{MHz}$. Some of TANS are working in this frequency range (e.g. Non Directional Beacon, NDB) and the impact of the emissions from WECS on them can be evaluated. In order to assess further TANS like Airport Surveillance Radar (ASR) a RF front end for the corresponding frequencies is presented in [7]. In the next chapter, the design of a RF front end for a frequency range from $150 \mathrm{kHz}$ until $30 \mathrm{MHz}$ will be discussed

\section{Receiver front end design}

As mentioned above, one requirement of the RF front end is the compatibility with the FMP. In [8] the main concepts of receivers are shown and discussed. The receiver types are separated in two groups - with and without frequency conversion. Due to the required compatibility with the signal digitizer board, the received signal should be converted to the intermediate frequency of $70 \mathrm{MHz}$. Therefore, the RF front end is designed as a heterodyne receiver.
In the first design step the structure scheme of a $\mathrm{RF}$ front end is developed. Its task is to select the frequency band and convert it to the intermediate frequency (IF). No further signal processing capabilities are needed. Those are carried out by other system components.

The main element of such a receiver is a mixer, which is a nonlinear three-port device with two in-ports and one out-port. An ideal mixer multiplies both input signals and outputs their product [9, p. 1363]. The received signal in frequency range of $150 \mathrm{kHz}$ till $30 \mathrm{MHz}$ should be converted to a frequency of $70 \mathrm{MHz}$. Hereinafter, the radio frequency (RF) signal is applied to one of the input ports, the local oscillator (LO) signal to the second input port and the IF is fixed to $70 \mathrm{MHz}$. The digitizer board samples the IF at 160 Msps so it remains in the first Nyquist zone. The mixer is in operation in up-converting mode. In order to obtain the IF signal the mixer multiplies the RF signal with the LO signal:

$$
\begin{aligned}
s_{I F}(t)= & \cos \left[\omega_{R F} t+\varphi(t)\right] \cdot \cos \left(\omega_{L O} t\right)= \\
= & \frac{1}{2} \cos \left[2 \pi\left(f_{R F}+f_{L O}\right) t+\varphi(t)\right]+ \\
& +\frac{1}{2} \cos \left[2 \pi\left(f_{R F}-f_{L O}\right) t-\varphi(t)\right]
\end{aligned}
$$

where

$\omega_{R F}-$ angular frequency of received RF signal

$\omega_{L O}$ - angular frequency of LO signal

$f_{R F}$ - frequency of received RF signal

$f_{L O}$ - frequency of LO signal

$\varphi(t)$ - phase of the received RF signal

$t$ - time.

The result of the structure scheme development is shown in Fig. 3.

The RF front end consists of a RF filter, an attenuator, a RF amplifier, two RF switches, a mixer, an IF filter, an IF amplifier, a LO filter and a LO amplifier. An antenna, which is resonant for the desired frequency range, should be connected to the RF front end input. The output of the receiver can be connected to the signal digitizer board. Furthermore, the function of each block in the structure is given. The RF filter limits the received signal spectrum to the range of $150 \mathrm{kHz}$ till $30 \mathrm{MHz}$. When it necessary the received signal can be attenuated by the attenuator and amplified by the RF amplifier, which can be patched in by the RF switches (automatic gain control, AGC). The mixer relocates the $\mathrm{RF}$ signal into the intermediate frequency. At the mixer output two products arise, according to (1). Due to non-linearity of the mixer at its output, intermodulation products can be obtained. In order to select the IF signal at the mixer's output, an IF filter is needed. Because of mixing and filtering losses an IF amplifier is used. For the mixing process, a LO signal is needed. 


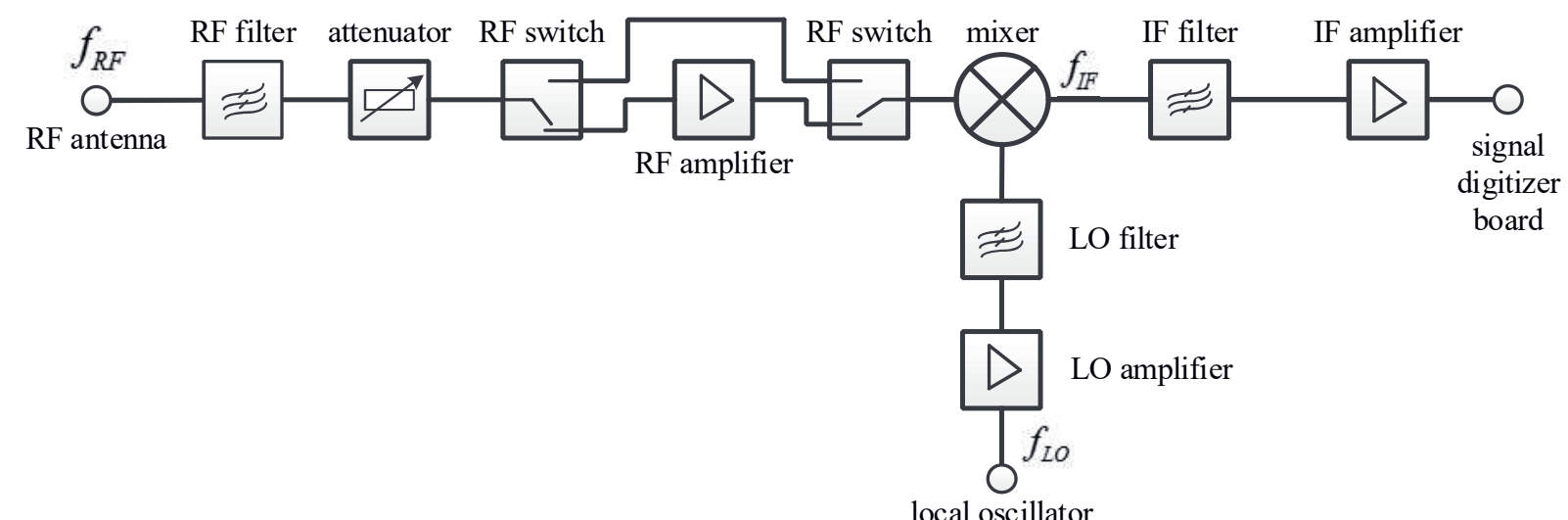

Fig.3. Structure of the RF front end

For this reason, the local oscillator input of the RF front end should be connected to the signal digitizer board. To ensure the desired sensitivity of the system, the mixer has to be driven by an LO signal of sufficient power level. As the LO amplifier creates harmonics due to its less than ideal linearity, the amplified LO signal has to be filtered to provide sufficient image rejection capabilities.

Using the characteristics of each chosen element, the characteristics such as third order intercept point, dynamic range and noise figure of the RF front end are determined.

Afterwards a prototype of the RF front end was build and tested. Fig. 4 shows the RF front end prototype. This prototype represents the structure shown on Fig. 3.

The RF front end prototype consists of evaluation boards, which are designed for every chosen element of the RF front end. The evaluation boards are mounted on a metal plate. Such design allows an evaluation of each element separately. Hereinafter, the whole prototype can be evaluated as well.

The successful evaluation of RF front end prototype leads to the final design of the receiver board as shown in Fig. 5.

The constructed RF front end board has a diameter of $180 \mathrm{~mm}$ and its weight is about 50 gram. Compared with convenient measurement equipment it has much smaller size and weight and can be integrated in the FMP.

\section{RF front end board evaluation}

After the discussion of the designed RF front end board, its characteristics are determined. For this reason, spectrum analyzer, vector network analyzer and a signal generator are needed. Using the vector network analyzer and the signal generator the conversion gain of the receiver is determined. Fig. 6 shows the conversion gain of designed receiver in dependence on frequency of the RF signal. It can be seen, that the conversion gain is nearly constant in the given frequency range.

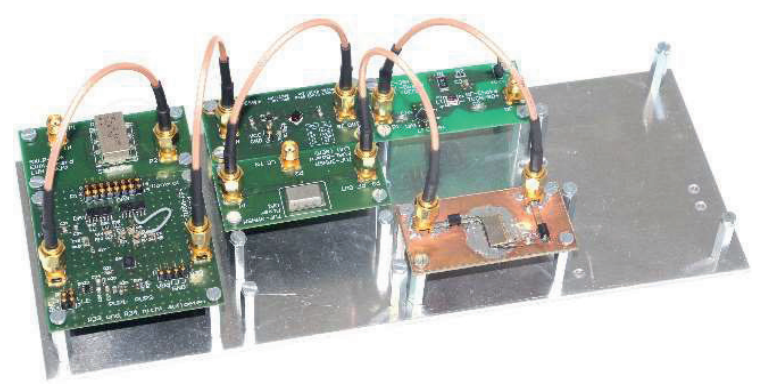

Fig. 4. Prototype of the receiver front end. Each element is positioned on its own evaluation board. Such design allows the evaluation of each element.

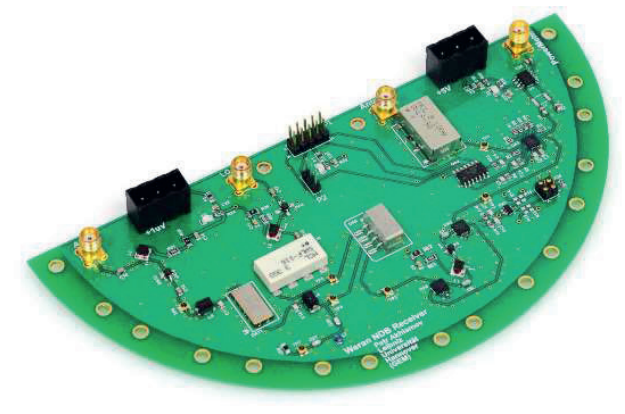

Fig. 5. The designed receiver front end. The form of the PCB allows easy integration into the FMP.

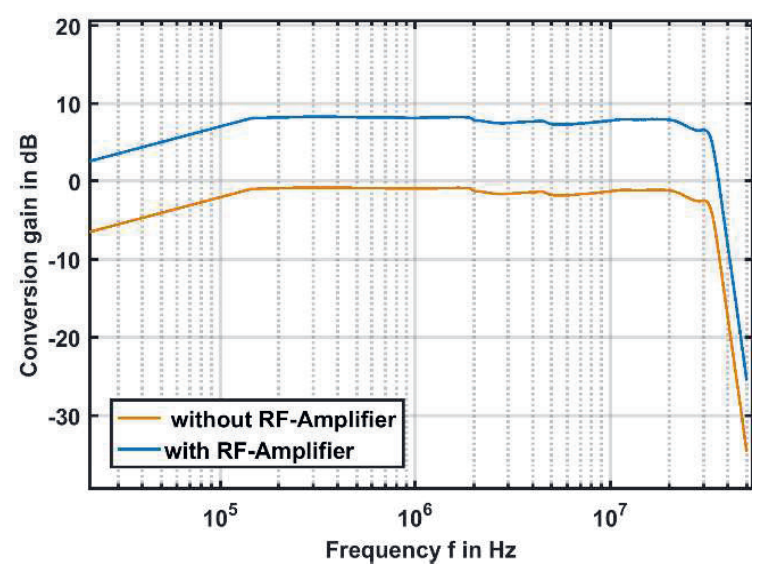

Fig. 6. Conversion gain of the RF front end. In frequency range from $150 \mathrm{kHz}$ till $30 \mathrm{MHz}$ the conversion gain is nearly constant. 
For the case, that the RF amplifier is used, the conversion gain is approx. $8 \mathrm{~dB}$ (see Fig. 6 , blue curve). Without the RF-amplifier a gain of approx. $-2 \mathrm{~dB}$ is achieved (see Fig.6, orange curve).

Furthermore, the third order intercept point of the receiver is determined using a two tones signal generator and a spectrum analyzer. Both, the measurement and the theoretical determination results, provide a good accordance. The third order intercept point of the RF front end board is approx. $-7 \mathrm{~dB}$, which limits the maximum signal strength of the RF front end board.

Hereinafter, the RF front end board was tested with one tone signal generator at frequency of $320 \mathrm{kHz}$, which simulates a NDB signal. The RF signal magnitude was set to $-20 \mathrm{dBm}$. Fig. 7 shows the spectral distribution of the obtained signal at RF front end output.

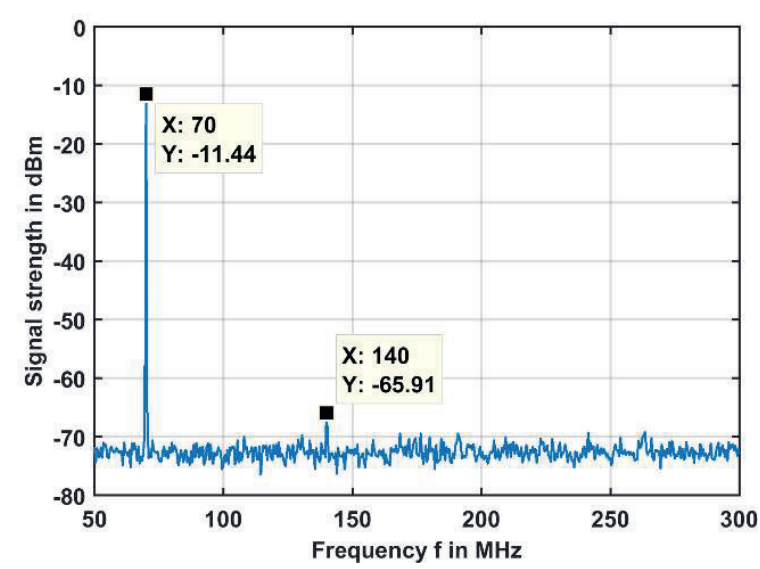

Fig. 7. Spectral distribution of the RF front end output signal. Beside the desired IF signal at $70 \mathrm{MHz}$, a second harmonic at $140 \mathrm{MHz}$ is present.

It can be seen, that the RF front end board converts the input RF signal into desired IF signal (70 MHz), amplifies it by approx. 8,56 dB and adds second harmonic at the frequency of $140 \mathrm{MHz}$. The magnitude of second harmonic is approx. $-65 \mathrm{dBm}$, which is much lower than the magnitude of usable IF signal. Thus, the spurious free dynamic range (SFDR) is $54.47 \mathrm{~dB}$. This measurement results promise satisfactory selectivity.

The measurement of the signal of a real NDB is also carried out. Due to weak level of the received NDB signal, only the ident tone (Morse code) was demodulated. This measurement proves good performance of designed RF front end board.

\section{Conclusion}

The number of wind energy converting systems (WECS) in Europe and especially in Germany has grown in last years. In case of installation of WECS near to terrestrial air navigation systems (TANS), the potential interactions between the WECS and the TANS must be examined. Therefore, measurements of electromagnetic (EM) fields are carried out. Currently, only measurements on the ground level near to WECS are feasible. Modern WECS typically have tower heights in excess of $100 \mathrm{~m}$ and a need to characterize their RF emissions at comparable heights above the ground arises.

In order to solve this metrological challenge, a modularly designed flying measuring platform (FMP) was developed. The FMP consists of a flight control unit, a signal digitizer board and a receiver front end with an antenna. The modular design of the FMP allows an easy re-equipment with a radio frequency receiver front end (RF front end) for the desired frequency range.

In this work, the development of a RF front end for the frequency range of $150 \mathrm{kHz}$ to $30 \mathrm{MHz}$ is presented. At first, the structure of the RF front end in consideration of technical requirements is developed. In order to assess the concept of the RF front end, evaluation boards were designed and tested. Satisfying evaluation results leaded to the design of the RF front end board, which can be easily integrated into the FMP. The result of the design is small weight and size RF front end with high performance in terms dynamic range and selectivity.

\section{Acknowledgment}

We would like to thank: T. Schrader, J. Rohde and M. Mihalachi (Department High Frequency and Electromagnetic Fields, PhysikalischTechnische Bundesanstalt, Braunschweig, Germany); B. Kokot, J. Fitschen, M. Mleczko and C. Reschka (Institute of Electrical Engineering and Measurement Technology, Leibniz Universität Hannover) for the close collaboration and inspiring discussions.

Furthermore, we acknowledge support by the joint project WERAN ("Wechselwirkung von Windenergieanlagen mit Radaranlage und terrestrischer Funknavigation") funded by the German Federal Ministry for Economic Affairs and Energy, FKZ: 0325644A. The scope of WERAN is the exploration of the interaction between wind turbines and safety-relevant terrestrial navigation systems. 


\section{References}

[1] UN/FCCC/CP/2015/L.9/Rev.1: Adoption of the Paris Agreement, Paris, 30 November to 11 December 2015

[2] IEC/CISPR 11:2015, Industrial, scientific and medical equipment - Radiofrequency disturbance characteristics - Limits and methods of measurement

[3] Technical Guidelines for Wind Turbines (FGW Guideline) Part 9:2014,Rev. 1, Determination of High Frequency Emissions from Renewable Power Generating Units

[4] IEC/CISPR/TR 16-2-5:2008, Specification for radio disturbance and immunity measuring apparatus and methods Part 2-5: In situ measurements of disturbing emissions produced by physically large equipment

[5] T. Schrader. J. Bredemeyer, Signal-inspace Measurements using Microcopters in Proc. $19^{\text {th }}$ International Flight Inspection Symposium (IFIS), Serbia, 2016.

[6] T. Schrader, J. Bredemeyer, M. Mihalachi, J. Rohde, T. Kleine- Ostmann, Concept and design of a UAS-based platform for measurements of RF signal-in-space in Advances in Radio Science, doi:10.5194/ars-14-1-2016

[7] J. Fitschen, S. Koj, J.Bredemeyer, H. Garbe, RF Front End for UAS-Aided Flight Inspection of Airport Surveillance Radars -in Proc. 11 th European Conference on Antennas and Propagation (EUCAP), Paris, France, 2017

[8] A. B. Carlson, P. B. Crilly, Communication Systems: An Introduction to Signals and Noise in Electrical Communication, 5th ed. McGraw-Hill, 2010.

[9] U. Tietze, C. Schenk, E. Schmid, Electronic Circuits: Handbook for Design and Applications, 2nd ed. Springer, 2008. 